\title{
A GENERALIZATION OF THE BORSUK AND BORSUK-ULAM THEOREMS
}

WALTER T. KYNER

The purpose of this paper is to give infinite dimensional generalizations of the classical Borsuk and Borsuk-Ulam theorems of algebraic topology. In addition, a topological property of compact linear transformations is established.

Let $E$ be an infinite dimensional real linear topological space with a bounded convex neighborhood of the origin. The fact that $E$ can be made into a normed linear space will be used, but the results depend on the given topology rather than on the chosen norm. All functions considered are continuous and are either defined on $V$, a convex body symmetric with respect to the origin, or on its boundary, $S$. The ranges of these functions are in $E$. A function is compact if the closure of its range is compact, and nonzero if the origin is not in its range. If $I$ is the identity map and $F$ is a compact map defined on $S$, then the function $f=I-F$ is a compact displacement of $S$. If $F(S)$ is finite dimensional, then $f=I-F$ is a finite displacement of $S$. And finally, a function $g$ is antipodal on $S$ if for all $x$ in $S, g(-x)$ $=-g(x)$.

The results of this paper follow from the fact that, after a consistent norm has been chosen, a compact antipodal map can be approximated by finite antipodal maps.

LEMma 1. If $f$ is a nonzero antipodal compact displacement of $S$, then given $\gamma>0$, there exists a nonzero antipodal finite displacement $g$, such that for all $x$ in $S$,

$$
\|f(x)-g(x)\|<\gamma .
$$

Proof. Since $f(S)$ is closed, $d=$ distance $(0, f(S))>0$. Let $\beta=1 / 2$ - min $(\gamma, d)$. By Leray-Schauder [3, Lemma 1], there exists a finite displacement $h$, such that for all $x$ in $S,\|h(x)-f(x)\|<\beta$.

$$
\begin{aligned}
&\|(1 / 2)[f(x)-f(-x)]-(1 / 2)[h(x)-h(-x)]\| \\
& \leqq(1 / 2)\|f(x)-h(x)\|+(1 / 2)\|f(-x)-h(-x)\|<\beta .
\end{aligned}
$$

Since $f(x)=-f(-x)$, if we set $g(x)=(1 / 2)[h(x)-h(-x)]$, we have

$$
\|f(x)-g(x)\|<\beta<\gamma, \text { and }
$$

Presented to the Society, April 15, 1955; received by the editors August 15, 1955 and, in revised form, February 1. 1956. 


$$
\|g(x)\| \geqq\|f(x)\|-\|f(x)-g(x)\| \geqq d-\beta>0 .
$$

Borsuk proved that the order of the origin with respect to a continuous antipodal nonzero map of the sphere (or of the boundary of any convex body) in Euclidean $n$-space is odd [1, p. 483]. A generalization of this to real Banach spaces was used by M. A. Krasnosel'skir in a study of nonlinear integral equations [2]. The generalization given here uses $\mathrm{E}$. Rothe's theory of topological order $[4 ; 5]$.

THEOREM 1. The order of the origin with respect to a nonzero antipodal compact displacement of $S$ is odd.

Proof. Let $u(f, S, 0)$ be the order of 0 with respect to $f(S)$. Take a nonzero antipodal finite displacement $h=I-H$ such that for all $x$ in $S,\|f(x)-h(x)\|<$ distance $(0, f(S))$. Then if $E^{m}$ is an $m$ dimensional subspace of $E$ containing $H(S)$, and if $S^{m-1}=S \cap E^{m}, h^{m} \mid S^{m-1}$, by Rothe, $u(f, S, 0)=u(h, S, 0)=u\left(h^{m}, S^{m-1}, 0\right)$. By Borsuk's theorem, $u\left(h^{m}, S^{m-1}, 0\right)$ is odd.

CoRollary 1. If $f$ is an antipodal compact displacement of $S$, then every half-line from the origin intersects the image of $S$.

Proof. Rothe has shown that if $0 \notin f(S)$ and $u(f, S, 0) \neq 0$, then every half-line from the origin intersects $f(S)$ [5, Theorem 7]. We have that either $0 \in f(S)$ or that $u(f, S, 0)$ is odd.

CoRollary 2. If $F$ is a compact map defined on $V$ such that $F$ is antipodal on $S$, then $F$ has a fixed point in $V$.

Proof. A fixed point of $F$ is a zero of $f=I-F$. If $0 \notin f(V)$, then $u(f, S, 0)=0[5$, Theorem 6]. The classical Borsuk-Ulam theorem implies that a continuous antipodal map of $S^{n}$, the sphere in $E^{n+1}$, into $E^{n}$ must map at least one pair of antipodal points onto the origin 1 , p. 486 , i.e. the space containing the range of the map must be of smaller dimension than the space containing the domain. In our infinite dimensional version, compactness of the image replaces the dimension decreasing hypothesis. Although the classical Borsuk and Borsuk-Ulam theorems are almost equivalent, the generalization of the former requires the heavy machinery of Rothe's theory, while the latter needs only a lemma analogous to Lemma 1 . (Here $E$ can be a complex linear space if the bounded convex neighborhood $U$ has the property that $x \in U$ implies that $e^{i \gamma} x \in U$ for all real $\gamma$.)

THEOREM 2. If $F$ is an antipodal map of $S$ into $E$ such that $F(S)$ is compact, then at least one pair of antipodal points of $S$ must map onto the origin. 
Proof. Since $F(S)$ is closed, if $0 \notin F(S)$, by the method of Lemma 1 , we can construct a nonzero antipodal map $G$ of $S$ into $E^{m} \subset E$. Take $E^{m+1} \supset E^{m}, S^{m}=S \cap E^{m+1}, G^{m+1}=G \mid S^{m}$.

$G^{m+1}: S^{m} \rightarrow E^{m}$, so by the finite dimensional theorem, there exists $z \in S^{m} \subset S$ such that $G^{m+1}(z)=G(z)=0$. Contradiction.

If we require only that $F$ be compact, we can only show that there exists a sequence of points in $S$ such that the limit of their images is 0 .

By considering linear transformations in a real separable Hilbert space into the Hilbert cube, we can show that for any non-negative integer $n$, there exists a compact linear transformation mapping exactly $n$ pairs of linearly independent antipodal points onto the origin. These examples led to the following result:

TheORem 3. In a reflexive Banach space (real or complex) a compact linear transformation has a nontrivial null space if and only if the image of the unit sphere is closed.

Proof. Let $B$ denote the unit ball and $S$ its boundary.

If $T$ is a compact linear transformation, then it is of course antipodal, and if $T(S)$ is closed, by Theorem $2, T$ has a nontrivial null space. (This can be proved directly without using algebraic topology.)

In a reflexive space, the image of the ball under any bounded linear transformation is closed [unpublished result of G. Weiss]. To finish the proof of our theorem, we need only show that $T(S)=T(B)$. Assume there exists $z \neq 0$ such that $T(z)=0$. Then if $y \in B$, we can pick a real number $\lambda$, such that $\|y+\lambda z\|=1$. Since $T(y)=T(y+\lambda z)$, we have $T(B)=T(S)$.

\section{BIBLIOGRAPHY}

1. Alexandroff-Hopf, Topologie, Berlin, 1935.

2. M. A. Krasnosel'skil, On the theory of completely continuous vector fields, Ukrain, Mat. Žurnal vol. 3 (1951) pp. 174-183.

3. J. Leray and J. Schauder, Topologie et équations fonctionnelles, Ann. École Norm. vol. 51 (1934) pp. 45-78.

4. E. Rothe, Zur Theorie der topologischen Ordnung und der Vektorfelder in Banachschen Räuman, Compositio Math. vol. 5 (1937) pp. 177-197.

5. - The theory of topological order in some linear topological spaces, Iowa State College Journal of Science vol. 13 (1939) pp. 373-390.

NORTHWESTERN UNIVERSITY 